\title{
Occurrence of low blood glucose concentrations during the afternoon in Type 2 (non-insulin-dependent) diabetic patients on oral hypoglycaemic agents: importance of blood glucose monitoring
}

\author{
M. Trovati, S. Burzacca, E. Mularoni, P. Massucco, F. Cavalot, L. Mattiello and G. Anfossi \\ Diabetes Unit, Department of Clinical and Biological Sciences, University of Turin, San Luigi Gonzaga Hospital, Orbassano, Turin, Italy
}

Summary. The European NIDDM Policy Group states that the lowest target for good control of Type 2 (non-insulin-dependent) diabetic patients is a blood glucose level $4.4 \mathrm{mmol} / \mathrm{l}$, both fasting and postprandially. The aim of this study is to evaluate the occurrence and temporal distribution of values under this target in the clinical records of 463 Type 2 diabetic patients, treated by diet or diet and oral hypoglycaemic agents, monitored for at least 2 years. The protocol includes blood glucose measurements after overnight fasting (08.00 hours), $120-150 \mathrm{~min}$ after breakfast (11.00 hours) and 120 and $240 \mathrm{~min}$ after lunch ( 14.00 and 16.00 hours). At least one blood glucose concentration of less than $4.4 \mathrm{mmol} / 1$ was presented by $42 \%$ of the patients. The only difference between patients showing and not showing glycaemic levels under this target was the higher percentage on oral hypoglycaemic agents in the first group ( $68.4 \%$ vs $56.9 \%, p=0.016)$. We considered 299 blood glucose profiles containing at least one value of less than $4.4 \mathrm{mmol} / \mathrm{l}$, observing that a) $46.9 \%$ of profiles from patients treated by diet alone and $68.7 \%$ of profiles from patients treated both by diet and oral hypoglycaemic agents presented the lowest blood glucose concentration at 16.00 hours $(p=0.002)$. b) No correlation existed between fasting blood glucose and values at 16.00 hours in profiles from diet-treated patients, whereas a negative correlation was present in patients on diet and oral hypoglycaemic agents, indicating that an excess of oral agents, administered to correct fasting hyperglycaemia. was the cause of the low glycaemic values in the afternoon. c) $37.9 \%$ of profiles on a diet and $83.3 \%$ of profiles on diet and oral agents showed fasting glucose concentrations $>6.7 \mathrm{mmol} / \mathrm{l}$, the upper limit of good control according to the European NIDDM Policy Group. This indicates that fasting hyperglycaemia does not exclude the occurrence of low glucose values throughout the day and that it is necessary to monitor blood glucose profiles.

Key words: Type 2 (non-insulin-dependent) diabetes mellitus, hypoglycaemia, oral hypoglycaemic agents, blood glucose profiles, monitoring protocols.
The targets of glycaemic control for Type 2 (non-insulindependent) diabetic patients are controversial. Recently, however, the European NIDDM Policy Group stated in a consensus view that patients should maintain fasting blood glucose levels between $4.4-6.7 \mathrm{mmol} / \mathrm{l}$, and postprandial levels between $4.4-8.9 \mathrm{mmol} / \mathrm{l}$, taking as an index of either acceptable or poor control, respectively, values $<7.8$ and $>7.8 \mathrm{mmol} / \mathrm{l}$ for fasting samples, and values $<10$ and $>10 \mathrm{mmol} / 1$ for postprandial samples [1]. The $4.4 \mathrm{mmol} / \mathrm{l}$ limit is due to the fact that lower concentrations could be dangerous in an older population frequently affected by vasculopathy, since values greater than the limits of chemical hypoglycaemia cause neurological abnormalities due to neuroglycopoenia and activate adrenergic glucose counterregulation [2]. The latter phenomenon is potentially dangerous as it can precipitate vascular complications of the disease owing to haemorbeological, haemodynamic and haemostatic changes, as it has been described during insulin-induced hypoglycaemia [3-9]. Since one of the main targets of Type 2 diabetes treatment is the prevention of angiopathy, it is particularly important to avoid low blood glucose concentrations. Data concerning severe hypoglycaemic episodes in Type 2 diabetic patients have been previously reported [10]. As far as we know, however, the prevalence of blood glucose concentrations under the $4.4 \mathrm{mmol} / 1$ target [1] has not been investigated.

The aims of the present study are to evaluate (1) the occurrence of blood glucose concentrations under the $4.4 \mathrm{mmol} / \mathrm{l}$ target in a cohort of Type 2 diabetic patients; (2) their temporal distribution and their relationship to treatment; (3) the usefulness of a clinical monitoring protocol involving daily blood glucose profiles. 
Table 1. Mean $\pm S E M$, ranges and intra-assay coefficients of variation obtained measuring venous blood glucose (ten determinations) and venous plasma glucose (ten determinations) on four venous blood samples obtained from a healthy volunteer before and after an i.v. insulin bolus. Venous blood glucose was measured by means of Reflolux II, venous plasma glucose by means of the Glucose Analyzer

\begin{tabular}{ll}
\hline $\begin{array}{l}\text { Venous blood glucose (mmol/l) } \\
n=10 \text { (Reflolux II) }\end{array}$ & $\begin{array}{l}\text { Venous plasma glucose (mmol/l) } \\
n=10 \text { (Glucose Analyzer) }\end{array}$ \\
\hline $4.9 \pm 0.03(4.7-5.1)(\mathrm{CV} 2.2 \%)$ & $5.1 \pm 0.03(5.0-5.2)$ (CV 1.7\%) \\
$4.1 \pm 0.03(4.0-4.3)$ (CV 2.3\%) & $4.2 \pm 0.03(4.1-4.3)$ (CV 1.9\%) \\
$3.0 \pm 0.03(2.9-3.1)$ (CV 3.3\%) & $3.2 \pm 0.01(3.1-3.2)$ (CV $1.2 \%)$ \\
$2.5 \pm 0.03(2.4-2.6)(\mathrm{CV} 3.4 \%)$ & $2.7 \pm 0.02(2.6-2.8)(\mathrm{CV} 2.0 \%)$ \\
\hline
\end{tabular}

Table 2. Mean \pm SEM, ranges and intra-assay coefficients of variation obtained measuring capillary blood glucose (four determinations from the same finger prick) and venous plasma glucose (four determinations from venous blood samples obtained simultaneously to finger pricks). The measurements were performed before and after an i. v. insulin bolus in an healthy volunteer. Capillary blood glucose was measured by means of Reflolux II, venous plasma glucose by means of the Glucose Analyzer

\begin{tabular}{ll}
$\begin{array}{l}\text { Capillary blood glucose (mmol/1) } \\
n=4 \text { (Reflolux II) }\end{array}$ & $\begin{array}{l}\text { Venous plasma glucose (mmol/l) } \\
n=4(\text { Glucose Analyzer) }\end{array}$ \\
\hline $4.6 \pm 0.08(4.4-4.7)(\mathrm{CV} \mathrm{3.4 \% )}$ & $4.5 \pm 0.03(4.4-4.6)(\mathrm{CV} 1.6 \%)$ \\
$4.0 \pm 0.05(3.9-4.1)(\mathrm{CV} 2.5 \%)$ & $4.0 \pm 0.04(3.9-4.1)(\mathrm{CV} 1.9 \%)$ \\
$2.5 \pm 0.04(2.4-2.6)(\mathrm{CV} 3.6 \%)$ & $2.5 \pm 0.03(2.4-2.5)(\mathrm{CV} 2.5 \%)$ \\
$2.1 \pm 0.04(2.0-2.2)(\mathrm{CV} \mathrm{3.3 \% )}$ & $2.0 \pm 0.02(2.0-2.0)(\mathrm{CV} 1.6 \%)$ \\
\hline
\end{tabular}

\section{Subjects and methods}

We examined the clinical records of a cohort of 463 Type 2 diabetic patients who were regularly attending the diabetic clinic of the San Luigi Gonzaga Hospital (Orbassano, Turin), being treated by diet alone or diet and oral hypoglycaemic agents (OHA). Type 2 diabetic patients on insulin treatment were not included in the cohort. The diagnosis of diabetes was made according to the criteria of the National Diabetes Data Group [11]. For a period of at least 2 years patients were submitted to follow-up examinations every $1-3$ months involving measurement of body weight, blood pressure, $\mathrm{HbA}_{\mathrm{lc}}$ (chromatographic method with microcolumns, Bio Rad, (Richmond, Calif., USA), mean value in a cohort of 200 healthy subjects $4.8 \pm 0.05 \%$, range $3.8-5.9 \%$ ) and capillary blood glucose profiles, measured by trained personnel, using a reflectance meter (Reflolux II, Boehringer, Mannheim, FRG). It is known that capillary glucose is slightly higher than venous glucose, and that blood glucose is slightly lower than plasma glucose [12], a fact to be kept in mind when comparing glucose measurements of different fluids. To assess the reliability of blood glucose measurements in the low range and to compare data obtained using the reflectance meter with those obtained using an established laboratory procedure, we determined the glucose concentrations both in the euglycaemic and low glycaemic range with the Glucose Analyzer (Beckman, Fullerton, Calif., USA) in plasma and with Reflolux II in blood. For this purpose, we used the same venous blood samples obtained from a healthy volunteer before and after an i. v. bolus injection of regular insulin, Actrapid HM (Novo, Copenhagen, Denmark $3.84 \mathrm{U} / \mathrm{m}^{2}$ ), an experimental model previously employed [6]. The results are shown in Table 1. Even if the intra-assay coefficients of variation (CV) are lower using the Glucose-Analyzer $(1.2-2.0 \%)$, they are also good with Reflolux II $(2.2-3.4 \%)$. In the $2.5-5.0 \mathrm{mmol} / \mathrm{l}$ range, a correlation of $r=0.99$ was obtained between glucose measurements carried out by the Glucose Analyzer and Reflolux II, evaluated by means of the linear regression formula.

The results from our laboratory are in agreement with those previously reported [13], demonstrating that Reflolux II is a very precise meter even in the low glycaemic range: in particular, the quoted study of Thai et al. [13] reported for low glucose values an intra-assay CV of $2.8 \%$ for Reflolux II, $2.5 \%$ for the Beckman Glucose Ana- lyzer, and a correlation coefficient between the reference method and Reflolux II of $r=0.99$. The same authors state that, when compared with two other widely used meters, only Reflolux II does not underestimate glucose values [13].

As we measured glucose concentrations in capillary blood, we also evaluated the intra-assay $\mathrm{CV}$ in the normal and low glycaemic range in this experimental condition, which could give rise to problems related to a more difficult blood collection. In six subjects, with blood glucose concentrations between $4.46 .6 \mathrm{mmol} / \mathrm{l}$, we determined the intra-assay CV in the measurement of capillary blood glucose by Reflolux II, taking into account the values of four different drops obtained from the same finger prick. The intra-assay CV ranged from $1.0 \%$ to $4.0 \%$. To determine the $\mathrm{CV}$ in the low glycaemic range, we submitted a healthy volunteer to an $\mathrm{i}$. v. insulin injection as previously described [6]. Four different finger pricks were made at different times during the test, obtaining four capillary blood drops from each prick to measure the capillary blood value. We compared the results with the plasma venous glucose determinations carried out on four venous blood samples obtained simultaneously with the finger pricks. Capillary blood glucose was measured by the Reflolux II, venous plasma glucose by Glucose Analyzer. The very high insulin sensitivity of the volunteer allowed us to measure the intra-assay $\mathrm{CV}$ also for glycaemic concentrations lower than those occurring in the clinical records of our patients (Table 2). Good intra-assay CV were also obtained with capillary blood ranging between $2.5 \%$ and $3.6 \%$ even if, owing to the experimental design, only four determinations could be made for each low glucose level.

In our study, one person was responsible for all the blood glucose measurements, performed at $08.00,11.00,14.00$ and 16.00 hours. Patients were asked to have breakfast between 08.30 and 09.00 hours, and lunch at 12.00 hours. The times were chosen to enable them to go home for their meals and to come back to the hospital for blood glucose measurements. This did not entail any appreciable increase in physical activity, as transport was provided for our patients by car or bus.

A diet containing $55 \%$ carbohydrates, $25 \%$ lipids, and $20 \%$ proteins was prescribed by a dietician. The patients were also advised not to eat snacks between the three major meals. Assessment of diabetic complications was performed annually.

As there were no diabeticservices in the area of the San LuigiGonzaga Hospital before 1984, patients often came to the specialized clinic after having been treated for many years by general practitioners. The early blood glucose profiles of their clinical records reflected therefore the treatment prescribed by their family doctors.

We evaluated: (1) the characteristics of the cohort: sex, age, age at diagnosis, diabetes duration, body mass index $\left(\mathrm{kg} / \mathrm{m}^{2}\right)$ and type of treatment; (2) the characteristics of the subgroups of the cohort presenting (Group A) or not presenting (Group B) at least one blood glucose concentration of less than $4.4 \mathrm{mmol} / \mathrm{l}$ (regarding $\mathrm{HbA}_{1 \mathrm{c}}$, we took into account the values corresponding to the profiles with low glycaemic concentrations in Group A patients); (3) the temporal distribution of blood glucose levels of less than $4.4 \mathrm{mmol} / 1$, and their relationship to the treatment.

\section{Statistical analysis}

Statistical analysis was carried out, when indicated, by analysis of variance for repeated measures, Student's $t$-test for paired and unpaired data, correlation coefficients and comparison between proportions [14]. Data in the text, tables and figures are expressed as mean $\pm S E M$.

\section{Results}

Table 3 shows the characteristics of the cohort investigated (463 Type 2 diabetic patients) and of the two groups: patients who presented at least one blood glucose concentra- 


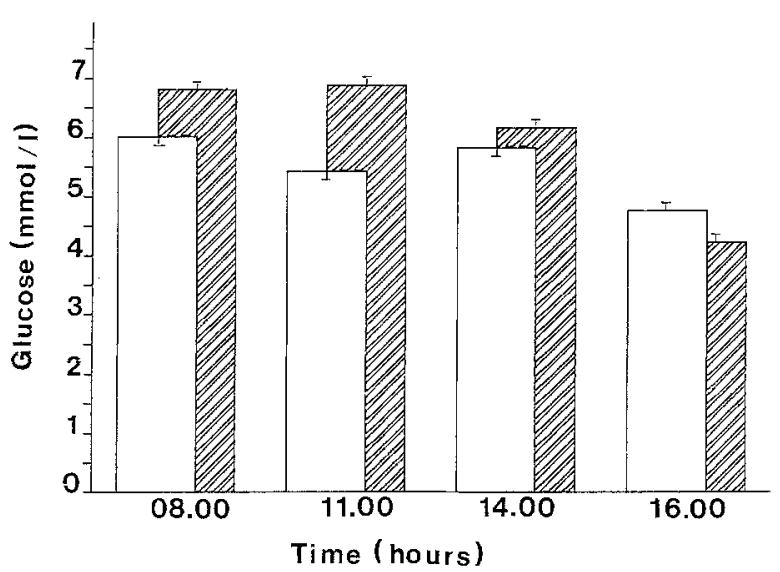

Fig. 1. Two hundred and ninety-nine blood glucose profiles (mean \pm SEM), containing at least one blood glucose concentration of less than $4.4 \mathrm{mmol} / 1$, measured in Type 2 (non-insulin-dependent) diabetic patients treated by diet alone ( $n=66$, white bars) or diet and oral hypoglycaemic agents $(n=233$, hatched bars). Compared to profiles from patients treated by diet and oral hypoglycaemic agents, profiles from diet-treated patients showed lower glucose concentrations at 08.00 and 11.00 hours $(p=0.003$ and $p=0.0005$, respectively), higher at 16.00 hours $(p=0.005)$. At 14.00 hours the difference was not significant

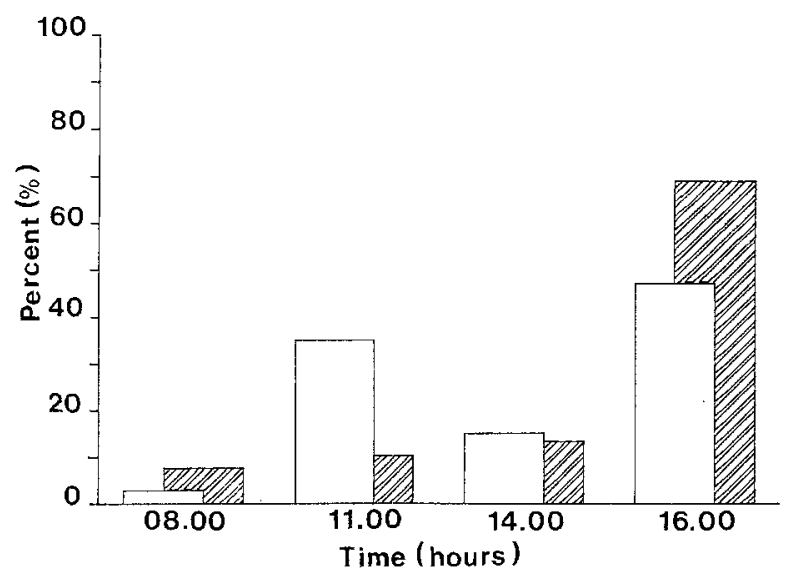

Fig. 2. Percent distribution of 299 blood glucose profiles, containing at least one blood glucose concentration of less than $4.4 \mathrm{mmol} / 1$, measured in Type 2 (non-insulin-dependent) diabetic patients treated by diet alone ( $n=66$, white bars) or diet and oral hypoglycaemic agents ( $n=233$, hatched bars), divided according to the hour of the day at which they presented the lowest blood glucose concentration. Compared to the percent distribution of profiles from patients treated by diet and oral hypoglycaemic agents, the percent distribution of profiles from diet-treated patients was higher at 11.00 hours $(p=0.0005)$, lower at 16.00 hours $(p=0.002)$. At 08.00 and 14.00 hours the difference was not significant

tion of less than $4.4 \mathrm{mmol} / \mathrm{l}(n=196)$ (Group A) and patients who never presented glucose concentrations lower than this target $(n=267)$ (Group B). The two groups do not differ regarding sex, age, age at diagnosis, diabetes $\mathrm{du}$ ration and body mass index. The only difference is the greater percentage of patients on OHA in Group A $(68.4 \%$ vs $56.9 \%, p=0.016)$. $\mathrm{HbA}_{1 \mathrm{c}}$ concentrations presented by Group A patients when their blood glucose profiles contained glycaemic values less than $4.4 \mathrm{mmol} / 1$ was $6.0 \pm 0.1 \%$, whereas $\mathrm{HbA}_{1 c}$ concentration of Group B patients was $7.6 \pm 0.1 \%$ ( $p=0.0005$ vs Group A).
Out of the 463 diabetic patients of the cohort, $42 \%$ showed blood glucose concentrations of less than $4.4 \mathrm{mmol} / \mathrm{l}: 12 \%$ of less than $3.3 \mathrm{mmol} / \mathrm{l}, 10 \%$ between 3.3 and $3.8 \mathrm{mmol} / \mathrm{l}$, and $20 \%$ between 3.8 and $4.3 \mathrm{mmol} / \mathrm{l}$. The majority of low blood glucose values were not reported as symptomatic by the patients, and objective signs were seldom noted by medical personnel. Of patients presenting glucose values of less than $3.3 \mathrm{mmol} / \mathrm{l}$, nine were treated with diet alone and 47 with diet and OHA. These patients did not differ from patients of Group B described in Table 3 regarding age $(59.8 \pm 1.3$ years $)$, sex $(\mathrm{M} / \mathrm{F}=0.9)$, body mass index $\left(29.4 \pm 0.6 \mathrm{~kg} / \mathrm{m}^{2}\right)$, age at diagnosis $(52.8 \pm 1.4$ years $)$ and diabetes duration $(7.0 \pm 0.9$ years $)$. The only striking difference was the percentage of patients treated with OHA ( $83.9 \%$ vs $56.9 \%, p=0.0005)$.

Since some patients of Group A presented more than one blood glucose profile with concentrations of less than $4.4 \mathrm{mmol} / \mathrm{l}$, we took 299 blood glucose profiles into account: $77.9 \%$ were measured in patients treated with diet and OHA. Figure 1 shows the mean values of blood glucose concentrations at different times of day, both in the 66 profiles from diet-treated and in 233 from diet- and OHA-treated patients. At 08.00 and 11.00 hours, values from patients treated with diet alone were lower than values from diet- and OHA-treated patients ( $p=0.003$ and $p=0.0005$ respectively); no difference was observed at 14.00 hours, whereas values at 16.00 hours were lower in patients treated with diet and OHA $(p=0.005)$. Both in profiles from diet-treated patients and in profiles from diet- and OHA-treated patients, the analysis of variance for repeated measures showed a highly significant difference between the different times $(p=0.0005)$, and paired Student's $t$-test showed that values at 16.00 hours are significantly lower than values at 08.00 hours $(p=0.0005$ in profiles from diet-treated and from dietand OHA-treated patients), at 11.00 hours ( $p=0.025$ in profiles from diet-treated and $p=0.0005$ in profiles from diet- and OHA-treated patients) and at 14.00 hours $(p=0.0005$ in profiles from diet- and from diet- and OHA-treated patients).

When the individual blood glucose profiles are taken into account in order to determine the time when the lowest blood glucose concentration occurs, Figure 2 shows that $47.0 \%$ of profiles from diet-treated and $68.7 \%$ of profiles from diet- and OHA-treated patients presented the lowest glycaemic value at 16.00 hours, the difference between diet-treated and diet- and OHAtreated being significant $(p=0.002$ ).

No correlation was observed between fasting blood glucose and the value obtained at 16.00 hours in profiles from diet-treated patients $(r=0.59$, NS), whereas a negative correlation was found in profiles from diet- and OHA-treated patients $(r=-0.263, p=0.0005)$. When the relationships between blood glucose levels during the day and the fasting levels were further investigated in profiles containing values of less than $4.4 \mathrm{mmol} / \mathrm{l}$, it was observed that $28.8 \%$ of profiles from diet-treated and $51.5 \%$ of profiles from diet- and OHA-treated patients presented a fasting blood glucose concentration between 6.7 and $7.8 \mathrm{mmol} / \mathrm{l}$, whereas $9.1 \%$ of profiles from diettreated and $31.8 \%$ of profiles from diet-and OHA-treated 


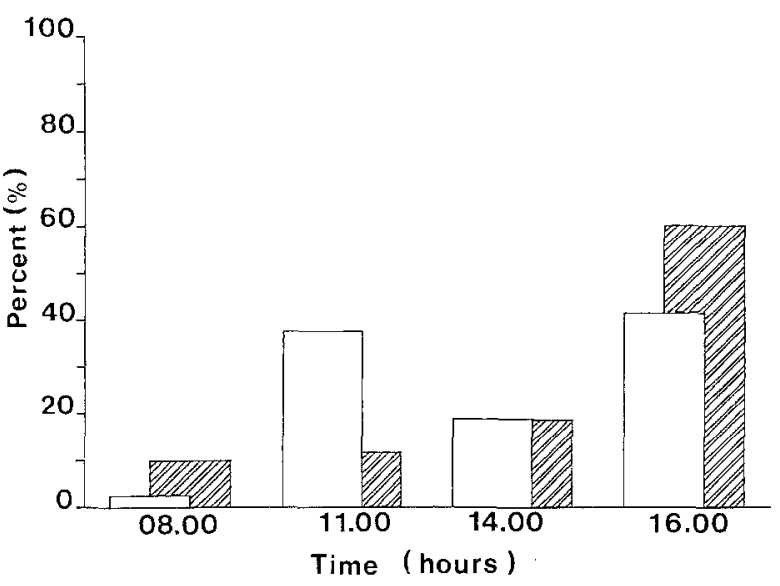

Fig. 3. Percent distribution of 382 blood glucose concentrations of less than $4.4 \mathrm{mmol} / \mathrm{l}$, contained in 299 blood glucose profiles, measured in Type 2 (non-insulin-dependent) diabetic patients treated by diet alone ( $n=80$, white bars) or diet and oral hypoglycaemic agents $(n=382$, hatched bars), divided according to the hour of the day at which they occurred. Compared to the percent distribution of blood glucose levels from patients treated by diet and oral hypoglycaemic agents, the percent distribution of blood glucose levels from diet-treated patients was lower at 08.00 hours $(p=0.057)$, higher at 11.00 hours $(p=0.0005)$, lower at 16.00 hours $(p=0.004)$. At 14.00 hours the difference was not significant

patients presented a fasting value $>7.8 \mathrm{mmol} / 1$. According to the European NIDDM Policy Group, fasting blood glucose concentrations between 6.7 and $7.8 \mathrm{mmol} / \mathrm{h}$ and $>7.8 \mathrm{mmol} / \mathrm{l}$ indicate that the blood glucose control is not good, but, respectively, either acceptable or poor [1]. Only $10 \%$ of profiles showing blood glucose concentrations under the target presented fasting levels of less than $4.4 \mathrm{mmol} / \mathrm{l}$. An elevated fasting concentration does not exclude low glucose values during the day: in our series, two profiles showing values of less than $4.4 \mathrm{mmol} / \mathrm{l}$ at 16.00 hours presented fasting values of 12.4 and $14.6 \mathrm{mmol} / 1$, respectively.

Since some of the 299 profiles taken into account contained more than one glycaemic level of less than $4.4 \mathrm{mmol} / \mathrm{l}$, we considered 382 glycaemic concentrations, 80 in profiles from diet-treated, 302 in profiles from dietand OHA-treated patients. Of these values, 63 were of less than $3.3 \mathrm{mmol} / \mathrm{l}$, nine in profiles from diet-treated, 54 in profiles from diet- and OHA-treated patients. All the values of less than $2.7 \mathrm{mmol} / \mathrm{l}(n=24)$ were in profiles from diet- and OHA-treated patients.

Figure 3 shows the temporal distribution of the glycaemic levels of less than $4.4 \mathrm{mmol} / \mathrm{l}$; OHA treatment increased the tendency for low glycaemic concentrations at 16.00 hours, in comparison with diet treatment alone $(60.0 \%$ vs $41.3 \%, p=0.004)$.

As far as hypoglycaemic treatment is concerned, $59.0 \%$ of profiles of Group A patients on OHA treatment presenting blood glucose values of less than $4.4 \mathrm{mmol} / \mathrm{l}$ were on sulphonylureas, $41.0 \%$ on pharmacological preparations containing sulphonylureas and biguanides. The most employed sulphonylurea was glibenclamide $(96.8 \%)$, the only biguanide employed was phenformin. Biguanides were not administered alone. No patient received a daily glibenclamide dose $>15 \mathrm{mg}$, or a daily
Table 3. Characteristics of a cohort of 463 Type 2 (non-insulin-dependent) diabetic patients and of the two subgroups presenting (Group A) and not presenting (Group B) glycaemic concentrations of less than $4.4 \mathrm{mmol} / \mathrm{l}$. Data are presented as mean $\pm \mathrm{SEM}$

\begin{tabular}{lcccl}
\hline & $\begin{array}{c}\text { Total cohort Group A } \\
(n=463)\end{array}$ & $\begin{array}{c}\text { Group B } \\
(n=196)\end{array}$ & $P$ value \\
$(n=267)$ & \\
\hline Age (years) & $59.0 \pm 0.5$ & $57.9 \pm 0.7$ & $59.8 \pm 0.7$ & NS \\
Sex (M/F) & 0.9 & 1.0 & 0.9 & NS \\
BMI (kg/m $\left.{ }^{2}\right)$ & $29.1 \pm 0.2$ & $29.2 \pm 0.4$ & $29.0 \pm 0.3$ & NS \\
Age at diagnosis (years) & $52.2 \pm 0.5$ & $51.3 \pm 0.8$ & $52.8 \pm 0.7$ & NS \\
Diabetes duration (years) & $7.1 \pm 0.3$ & $6.6 \pm 0.4$ & $7.3 \pm 0.4$ & NS \\
Treatment: diet (\%) & 38.4 & 31.6 & 43.1 & 0.016 \\
diet + OHA (\%) & 61.5 & 68.4 & 56.9 & 0.016 \\
\hline
\end{tabular}

Significance refers to differences between Group A and Group B. $\mathrm{OHA}=$ oral hypoglycaemic agents

phenformin dose $>75 \mathrm{mg}$. Doses greater than $5 \mathrm{mg}$ glibenclamide or $2.5 \mathrm{mg}$ glibenclamide and $25 \mathrm{mg}$ phenformin were administered as divided doses. Regarding the timing of $\mathrm{OHA}$ administration in patients presenting glucose values of less than $4.4 \mathrm{mmol} / \mathrm{l}, 52.9 \%$ of profiles were from OHA before breakfast, lunch and supper treatment; $13.7 \%$ before lunch and supper; $12.9 \%$ before breakfast and supper; $7.5 \%$ before lunch; $7.0 \%$ before breakfast and lunch; $4.0 \%$ before breakfast; $2.2 \%$ before supper. $58.5 \%$ of OHA doses before breakfast were low (2.5 $\mathrm{mg}$ glibenclamide or $1.75 \mathrm{mg}$ glibenclamide and $12.5 \mathrm{mg}$ phenfor$\mathrm{min}$ ). When glibenclamide is employed, a reasonable schedule, owing to the pharmacokinetic characteristics of the drug, is before breakfast, and, if necessary, also before supper. $16.7 \%$ of profiles containing at least one blood glucose value of less than $4.4 \mathrm{mmol} / \mathrm{l}$ presented this kind of schedule, with four glycaemic concentrations of less than $3.3 \mathrm{mmol} / \mathrm{l}$. All schedules of OHA administration showed the majority of blood glucose concentrations of less than $4.4 \mathrm{mmol} / \mathrm{l}$ occurring at 16.00 hours: $77.5 \%$ in profiles with OHA before breakfast, lunch and supper; $83.9 \%$ in profiles with $\mathrm{OHA}$ before lunch and supper; $72.4 \%$ in profiles with OHA before breakfast and supper; $70.6 \%$ in profiles with OHA before lunch; $81.3 \%$ in profiles with OHA before breakfast and lunch; $66.7 \%$ in profiles with OHA before breakfast; $80.0 \%$ in profiles with OHA before supper. Differences between these percentages were not significant.

\section{Discussion}

This study shows that (1) Type 2 diabetic patients can frequently present blood glucose values under the target stated by the European NIDDM Policy Group vis. $4.4 \mathrm{mmol} / 1$ [1]; (2) these episodes occur frequently in the afternoon; (3) oral hypoglycaemic treatment is responsible for a large number of the low glycaemic concentrations; (4) to detect blood glucose levels under the target, it is useful to perform blood glucose profiles, or, at least, to check the blood glucose concentrations in the afternoon.

The study was carried out using a reflectance meter which is precise even in the low glycaemic range [13]. The glycaemic targets recommended by the European Policy Group refer to venous blood measurements [1], whereas our study has been carried out on capillary blood em- 
ploying a reflectance meter, as this is usually used in the clinical monitoring protocols and strongly recommended for self-monitoring diabetic patients. As we previously discussed, a perfect comparison between the glucose determinations carried out in different fluids is not possible. We can reasonably exclude, however, that the presence of low glycaemic levels has been overestimated in this study, since the venous blood glucose values - taken into account by the European Policy Group - are slightly lower than capillary glucose values [12].

No severe hypoglycaemic episodes with loss of consciousness or requiring i.v. glucose administration or a glucagon injection were observed in our series. The great majority of low glycaemic levels were not reported by the patients, partly because they were asymptomatic, partly because symptoms were not recognized or erroneously ascribed to cardiovascular or neurological causes. This observation is in keeping with a study showing that only $45 \%$ of Type 2 diabetic patients experiencing hypoglycaemic symptoms spontaneously report them to the diabetic clinic [15], and underlines the need for comprehensive patient education to increase the awareness of the occurrence of mild hypoglycaemia. Specific questions on this subject should be included in each medical examination of Type 2 diabetic patients.

When we consider the possible causes of blood glucose concentrations under the $4.4 \mathrm{mmol} / 1$ target in our study, it is necessary to distinguish between patients on diet treatment and patients on diet and OHA treatment. The patients on a diet were often obese subjects who developed diabetes mellitus. When placed on a diet, they showed a marked improvement in blood glucose control, but not a complete normalization. Their fasting blood glucose concentrations remained slightly elevated, whereas the postprandial decrease of blood glucose in the afternoon could indicate the presence of an exaggerated and delayed insulinaemic response to food. The blood glucose decrease observed after breakfast might also be interpreted in this light.

The trend toward lowest glycaemic concentrations of the profile in the afternoon is, however, much more evident in patients on diet and OHA treatment. These patients presentedblood glucose values higher than patients on diet alone after an overnight fast and after breakfast and lower $4 \mathrm{~h}$ after lunch. A pharmacological effect could therefore be supposed. Since in our survey glibenclamide was the most widely used of the sulphonylureas, one cannot rule out the possible responsibility of the divided dose schedule, frequently employed by general practitioners in Italy: this schedule is tempting, since the plasma concentrations of the drug increase after each administation, and this increase influences the glycaemic response to the meal consumed immediately after [16]. In Italy, breakfast is usually light, whereas the midday meal is the largest of the day: it is therefore not surprising that a schedule widely used by family doctors consists in giving small doses of OHA (i. e. half a tablet) before breakfast and/or supper and a larger dose (i.e. one tablet) before the midday meal. This type of prescription reflects more the amount of food to be eaten immediately after than the pharmacokinetic properties of glibenclamide: this drug, in fact, shows a relatively short half-life [16] but a slowly equilibrating deep compartment [17]. On repeated dosing, there is a potential risk of multiple overlaps of the drug action [17]. In particular, it could further aggravate the tendency of Type 2 diabetic patients-already present when they are on a diet alone - to have low blood glucose concentrations in the afternoon. Unfortunately, the last phenomenon is not easy to detect, and, subsequently, correct. In fact, patients are frequently unaware of mild hypoglycaemic episodes and do not report them to the physician; furthermore, fasting blood glucose, the only level usually checked by general practitioners, is not indicative of low glycaemic concentrations during the day, as this study shows.

Fasting blood glucose concentrations are certainly extremely important to assess the "severity" of diabetes [18]. In our study, for instance, fasting levels of OHA-requiring patients, in spite of the treatment, are higher than levels of patients on a diet alone. Fasting blood glucose, however, is not enough to evaluate whether the treatment regimen is able to avoid low blood glucose values during the day. For instance, in our study $90 \%$ of profiles showing glycaemic levels under the target presented euglycaemic or hyperglycaemic fasting concentrations. Furthermore, in OHAtreated patients, we observed a negative correlation between fasting blood glucose and values $4 \mathrm{~h}$ after the midday meal. Since this phenomenon does not exist in patients on a diet alone, it is clearly due to a pharmacological effect, indicating that, in order to improve fasting hyperglycaemia, an excess of OHA has been prescribed, causing low blood glucose concentrations in the afternoon. To verify the safety of OHA treatment, blood glucose profiles should be performed in Type 2 diabetic patients; at least a late afternoon value should be regularly checked. The risk of repeated low glucose values must be carefully considered in an older population potentially affected by atherosclerotic lesions, to avoid the iatrogenic effects of neuroglycopoenia and of catecholamine-mediated vascular damage [2-9]. In this light, the fasting and postprandial glycaemic targets of the European Policy Group [1] should be considered in an integrate view, and not separately. Actually, an abuse of oral agents aimed at attaining "good" fasting control could be dangerous for the patient if it is the cause of repeated low blood glucose concentrations in the afternoon. A higher fasting level must be accepted on these occasions. Oral agents should be employed with a greater caution in clinical practice. Unfortunately, as Tattersall observed, "Tablets are deceptively easy to prescribe" [19].

\section{References}

1. Alberti KGMM, Gries FA (1988) Management of non-insulindependent diabetes mellitus in Europe: a consensus view. Diab Med 5: 275-281

2. De Feo P, Gallai V, Mazzotta G et al. (1988) Modest decrements in plasma glucose concentration cause early impairment in cognitive function and later activation of glucose counterregulation in the absence of hypoglycaemic symptoms in normal man. $J$ Clin Invest 82: 436-444

3. Hilsted J, Bonde-Petersen F, Norgaard MB, Greniam M, Christiansen NJ, Parving HH, Suzuki M (1984) Haemodynamic 
changes in insulin-induced hypoglycaemia in man. Diabetologia 26: $328-332$

4. Fisher BM, Baylis PH, Frier BM (1987) Plasma oxytocin, arginine vasopressin and atrial natriuretic peptide responses to insulin-induced hypoglycaemia in man. Clin Endocrinol 26: 179185

5. Trovati M, Anfossi G, Cavalot F et al. (1986) Studies on mechanisms involved in hypoglycaemia-induced platelet activation. Diabetes 35: 818-825

6. Trovati M, Massucco P, Mularoni E, Cavalot F, Anfossi G, Mattiello L, Emanuelli G (1988) Insulin-induced hypoglycaemia increases plasma concentrations of angiotensin II and does not modify atrial natriuretic polypeptide secretion in man. Diabetologia 31: 816-820

7. Kishikawa H, Takeda H, Kiyota S et al. (1987) Role of $\alpha 2$ adrenergic receptor in platelet activation during insulin-induced hypoglycaemia in normal subjects. Diabetes 36: 407-412

8. Takeda $H$, Kishikawa $H$, Shinohara $M$ et al. (1988) Effect of $\alpha 2$ adrenoceptor antagonist on platelet activation during insulininduced hypoglycaemia in Type 2 (non-insulin-dependent) diabetes mellitus. Diabetologia 31: 657-663

9. Frier BM, Hilsted J (1985) Does hypoglycaemia aggravate the complications of diabetes? Lancet II: $1175-1177$

10. Ferner RE, Neil H (1988) Sulphonylureas and hypoglycaemia. Br Med J 296: 949-950

11. National Diabetes Data Group (1979) Classification and diagnosis of diabetes mellitus and other categories of glucose intolerance. Diabetes 28: 1039-1057

12. Foster DW (1991) Diabetes Mellitus. In: Wilson JD, Braunwald E, Isselbacher KJ, Petersdorf RG, Martin JB, Fauci AS, Root RK (eds) Harrison's principles of internal medicine, 12th edn. Mc Graw Hill, New York, pp 1739-1759
13. Thai AC, Ng WF, Lui KF, Yeo PPB (1989) Three new glucose reflectance meters Diascan, Glucometer II, and Reflolux II. Diab Res Clin Pract 7: 75-81

14. Glantz SA (1987) Primer of Biostatistics. Mc Graw Hill, New York

15. Jennings AM, Wilson RM, Ward JD (1989) Symptomatic hypoglycaemia in NIDDM patients treated with oral hypoglycaemic agents. Diab Care 12: 203-208

16. Matsuda A, Kuzuya T, Sugita Y, Kawashima K (1983) Plasma levels of glibenclamide in diabetic patients during its routine clinical administration determined by specific radioimmunoassay. Horm Metab Res 15: 425-428

17. Balant L, Zahnd GR, Weber F, Fabrie J (1977) Behaviour of glibenclamide on repeated administration to diabetic patients. Eur J Clin Pharmacol 11: 19-25

18. Holman RR, Turner RC (1988) Optimizing blood glucose control in Type 2 diabetes: an approach based on fasting blood glucose measurements. Diab Med 5: 582-588

19. Tattersall RB (1984) Diabetes in the elderly - a neglected area? Diabetologia 27: 167-173

Received: 22 November 1990

and in final revised form: 13 May 1991

Prof. M. Trovati

Dipartimento di Scienze Cliniche e Biologiche

dell' Universita' di Torino

Ospedale San Luigi Gonzaga

I-10043 Orbassano (Torino)

Italy 